\title{
Trans-sonic propeller substage
}

\author{
S. B. POPOV* and M. E. PROKHOROV \\ Sternberg Astronomical Institute, Moscow State University, Moscow 119992, Russia
}

(Received 11 November 2004)

\begin{abstract}
We follow the approach used by Davies and Pringle and discuss the trans-sonic substage of the propeller regime. This substage is intermediate between the supersonic and subsonic propeller substages. In the trans-sonic regime an envelope around a magnetosphere of a neutron star passes through a kind of reorganization process. The envelope in this regime consists of two parts. In the bottom part, turbulent motions are subsonic. Then at some distance $r_{\mathrm{s}}$ the turbulent velocity becomes equal to the sound velocity. During this substage the boundary $r_{\mathrm{s}}$ propagates outwards until it reaches the outer boundary, and so the subsonic regime starts. We found that the trans-sonic substage is unstable; so the transition between supersonic and subsonic substages proceeds on a dynamic timescale. For realistic parameters this time is in the range from weeks to years.
\end{abstract}

Keywords: Compact objects; Isolated neutron stars; Evolution

\section{Introduction}

The observational appearances of a neutron star (NS) are mainly determined by its interaction with the surrounding plasma. The following main stages (regimes) can be distinguished (see a very detailed description in [1] or [2]).

(i) Ejector. At this stage, plasma is swept away by low-frequency electromagnetic radiation or/and by a flow of relativistic particles. Matter is stopped further than the so-called light cylinder radius $r_{1}$.

(ii) Propeller. If an NS is in the propeller regime, then matter can penetrate inside $r_{1}$, but it is stopped by a rapidly rotating magnetosphere of the NS.

(iii) Accretion. Finally the NS is slowed down and the centrifugal barrier disappears; so, if matter cools sufficiently rapidly, then it can fall on to the surface of the compact object.

Normally an NS is born at the stage of ejection (a radio pulsar is a classical example of an NS at the ejector stage). Then, as the spin period increases, the NS passes through the propeller and accretor stages. For NSs with large (greater than $400 \mathrm{~km} \mathrm{~s}^{-1}$ ) spatial velocities, another stage, namely the georotator, can appear.

\footnotetext{
*Corresponding author. Email: polar@sai.msu.ru
} 
(a)

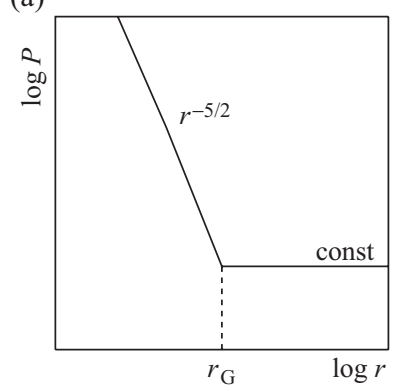

(b)

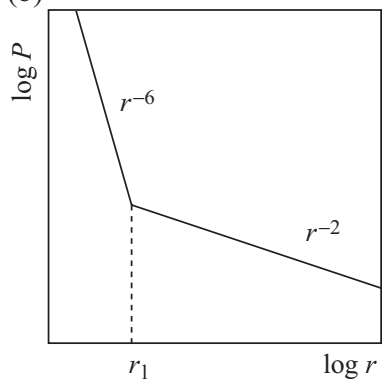

Figure 1. Pressure versus radius: (a) external pressure; (b) internal pressure.

In a simplified model it is possible to define transitions between different stages by comparing external and internal pressures (figure 1). The external pressure can be roughly approximated as the ram pressure of a flow of the interstellar medium (far from the NS) or as the pressure of matter falling on to the NS in its gravitational field (for distances smaller than the so-called gravitational capture radius $r_{\mathrm{G}}$ ). The internal pressure inside the light cylinder $r_{1}$ can be estimated as a pressure connected with the magnetic field of an NS.

In this paper we discuss the propeller stage. Its existence was recognized long ago (initially by Shvartsman [3] and later by Illarionov and Sunyaev [4]). However, this stage is still not well understood. Here we introduce and discuss a new substage of this regime of the magnetorotational evolution of NSs.

\section{General features of the propeller stage}

Davies et al. [5] and Davies and Pringle [6] noted that at the propeller stage there can be a significant energy release at the magnetospheric boundary. The energy can be high enough to form a kind of turbulent quasistatic atmosphere. These workers distinguished three substages of the propeller regime.

(i) The very rapid rotator substage is given by

$$
c_{\mathrm{s}}\left(r_{\mathrm{in}}\right) \approx r_{\mathrm{in}} \Omega \gg v_{\mathrm{ff}},
$$

where $c_{\mathrm{s}}$ is the sound velocity, $\Omega=2 \pi / P$ is the spin frequency, and $v_{\mathrm{ff}}$ is the free-fall velocity.

(ii) The supersonic propeller substage is given by

$$
r_{\text {in }} \Omega \gg c_{\mathrm{s}}\left(r_{\text {in }}\right) .
$$

(iii) The subsonic propeller substage is given by

$$
r_{\text {in }} \Omega \ll c_{\mathrm{s}}\left(r_{\text {in }}\right)
$$

and

$$
v_{\mathrm{t}}(r)<c_{\mathrm{s}}(r) \text { in } r_{\text {in }}<r<r_{\text {out }}=r_{\mathrm{G}},
$$

where $r_{\text {in }}$ and $r_{\text {out }}$ are the internal and external radii respectively of an envelope and $v_{\mathrm{t}}$ is the turbulent velocity. 
We shall not discuss the substage of the very rapid propeller here. Mainly we focus on the supersonic and subsonic substages and on the transition between them. The supersonic regime can be considered a classical propeller where accretion is impossible owing to a centrifugal barrier. At the subsonic stage the magnetospheric (Alfven) radius is smaller than the corotation radius $r_{\mathrm{c}}$. Accretion does not start because the temperature is too high (see original discussion given by Davies and Pringle [16] and later proposals by Ikhsanov [7]).

Braking laws (for the spin-down) are different at different substages. In the supersonic regime the energy loss rate does not depend on the spin period [6]; however, different formulae for the spin-down at this stage were suggested; see a list for example in [8]. In the subsonic substage the spin-down is always slower: $P \propto t$.

\section{Why does the intermediate regime exist?}

In general supersonic and subsonic regimes cover all possible values of the rotation velocity $\Omega=2 \pi / P$. The supersonic propeller formally operates until $r_{\text {in }}^{\text {super }} \Omega \geq c_{\mathrm{s}}\left(r_{\text {in }}^{\text {super }}\right)$. Here $r_{\text {in }}^{\text {super }}=r_{\mathrm{G}}^{2 / 9} r_{\mathrm{M}}^{7 / 9}[6]$, where $r_{\mathrm{M}}=\left\{\mu^{2} / \dot{M}(2 G M)^{1 / 2}\right\}^{2 / 7}$ is the magnetospheric (Alfven) radius.

The subsonic regime occurs when

$$
r_{\text {in }}^{\text {sub }} \Omega \leq c_{\mathrm{s}}\left(r_{\text {in }}^{\text {sub }}\right) \text { and } v_{\mathrm{t}}(r) \leq c_{\mathrm{s}}(r) \text { for } r_{\text {in }}^{\text {sub }} \leq r \leq r_{\text {out }}=r_{\mathrm{G}} \text {, }
$$

where $r_{\text {in }}^{\text {sub }}=r_{\mathrm{M}}[6]$. For the subsonic stage, $v_{\mathrm{t}}\left(r_{\mathrm{in}}^{\mathrm{sub}}\right) \approx r_{\mathrm{in}}^{\mathrm{sub}} \Omega$ and $v_{\mathrm{t}} / c_{\mathrm{s}} \propto r^{1 / 3}$; therefore this regime is valid for $r_{\text {in }}^{\text {sub }} \Omega \leq c_{\mathrm{s}}\left(r_{\text {in }}^{\text {sub }}\right)\left(r_{\text {in }}^{\text {sub }} / r_{\mathrm{G}}\right)^{1 / 3}$.

Note the following properties of the stages.

(i) At both stages the internal radii $r_{\text {in }}$ of an envelope do not depend on $\Omega$, and $r_{\text {in }}^{\text {super }}>r_{\text {in }}^{\text {sub }}$ always.

(ii) The structures of the envelopes for the two substages are different.

(iii) It is easy to check that the end of the supersonic substage and the beginning of the subsonic substage both correspond to

$$
\begin{aligned}
& \Omega=(2 G M)^{1 / 2} r_{\mathrm{M}}^{-7 / 6} r_{\mathrm{G}}^{-1 / 3}, \\
& P \approx 1.15 \times 10^{4} \mathrm{~s} \mu_{30}^{2 / 3} v_{6}^{1 / 3} \rho_{-24}^{-1 / 3} .
\end{aligned}
$$

Even in the frame of the Davies-Pringle model an intermediate regime during which the structure of the envelope is reorganized is inevitable. We call this intermediate stage the trans-sonic propeller. As we show below, this is a short non-equilibrium episode in the life of an NS, and the spin frequency does not change significantly during this reorganization process.

\section{Trans-sonic propeller substage}

Let us consider the structure of a quasistationary envelope at the intermediate trans-sonic propeller substage. In the atmosphere around such an NS, $c_{\mathrm{s}}(r) \approx v_{\mathrm{ff}}[6]$. (This condition is valid also for the supersonic and subsonic propeller substages.) Processes in the lower part of the atmosphere are similar to those in the subsonic substage:

$$
v_{\mathrm{t}}\left(r_{\mathrm{in}}\right) \approx r_{\mathrm{in}} \Omega<c_{\mathrm{s}}
$$


If the envelope (atmosphere) is adiabatic, then for this bottom part of it the polytropic index is equal to $n=3 / 2$ and

$$
\rho(r) \propto r^{-3 / 2}, \quad p(r) \propto r^{-5 / 2} .
$$

We assume following Davies and Pringle [6] that the rotational energy of the NS is dissipated at the magnetospheric boundary and that it is transported outwards by turbulence. For such assumptions we have

$$
v_{\mathrm{t}}(r) \propto r^{-1 / 6}
$$

and the turbulent Mach number is

$$
\mathcal{M}_{\mathrm{t}}(r) \equiv \frac{v_{\mathrm{t}}(r)}{c_{\mathrm{s}}(r)} \propto r^{1 / 3} .
$$

For $\mathcal{M}_{\mathrm{t}}<1$, that is while $r<r_{\mathrm{s}}$ where $r_{\mathrm{s}}$ is the boundary between the two parts of the envelope $\left(r_{\text {in }}<r_{\mathrm{s}}<r_{\mathrm{G}}\right)$, the structure of the envelope is not changed. For large radii, turbulence becomes supersonic. Small-scale shock waves are formed and they quickly dissipate part of the energy, so that the turbulent velocity decreases to the sound velocity. In the range $r_{\mathrm{s}}<r<r_{\mathrm{G}}$ the envelope structure is different from the bottom part:

$$
\begin{gathered}
\mathcal{M}_{\mathrm{t}}(r) \approx 1, \\
\rho(r) \propto r^{-1 / 2}, \quad p(r) \propto r^{-3 / 2} .
\end{gathered}
$$

In the outer part of the envelope, physical conditions are similar to those in the supersonic substage.

To determine the parameters of the whole atmosphere, it is necessary to calculate the position of the boundary between the two parts of the envelope, $r_{\mathrm{s}}$, and the position of the inner boundary of the bottom part, $r_{\text {in }}$ (during the transition it decreases from $r_{\mathrm{G}}^{2 / 9} r_{\mathrm{M}}^{7 / 9}$ to $r_{\mathrm{M}}$ ). To do this, it is necessary to solve the following system of equations:

$$
\begin{aligned}
\frac{\mu^{2}}{8 \pi} \frac{1}{r_{\text {in }}^{6}} & =\frac{1}{2} \frac{\dot{M} v_{\infty}}{4 \pi r_{\mathrm{G}}^{2}}\left(\frac{r_{\mathrm{G}}}{r_{\mathrm{s}}}\right)^{3 / 2}\left(\frac{r_{\mathrm{s}}}{r_{\mathrm{in}}}\right)^{5 / 2}, \\
\Omega r_{\mathrm{in}}^{7 / 6} r_{\mathrm{s}}^{-1 / 6} & =\left(\frac{2 G M}{r_{\mathrm{s}}}\right)^{1 / 2} .
\end{aligned}
$$

However, the system is degenerate and each equation can be reduced to $r_{\mathrm{s}} \propto r_{\text {in }}^{-7 / 2}$. If the equation

$$
\frac{\mu^{2}}{8 \pi}=\frac{1}{2} \frac{\dot{M} v_{\infty}}{4 \pi r_{\mathrm{G}}^{1 / 2}} \frac{(2 G M)^{3 / 2}}{\Omega^{3}}
$$

is fulfilled, then the system is compatible, that is, for all $r_{\text {in }}$ in the range $r_{\mathrm{M}}<r_{\text {in }}<r_{\mathrm{M}}^{7 / 9} r_{\mathrm{G}}^{2 / 9}$, there is some $r_{\mathrm{s}}$ that is a solution of equation (13).

The compatibility condition is fulfilled at the end of the supersonic substage. Later (during the transition) at any given moment (for any $\Omega$ ) the left-hand side of equation (14) is smaller than the right-hand side. This means that the magnetospheric pressure and the envelope pressure have the same dependences on $r_{\text {in }}$, but the latter is always larger (the first equation of the system (13)).

During the trans-sonic stage the period is not changing significantly (a typical value is determined by equation (6)); so in terms of the rotational evolution the subsonic substage nearly immediately follows the supersonic substage. The spin-down law for the trans-sonic propeller is the same as for the subsonic regime. 
The energy release during the transition stage is negligible. Estimates for realistic isolated NS parameters give a value $\Delta E \lesssim 10^{30} \mathrm{erg}$.

\section{Discussion}

We wish to note that calculations similar to those presented above are just rough estimates. There are several reasons for that.

The first is connected with uncertainties in many parameters, and even in their determinations. For example the accretion rate is usually taken as $\dot{M}=\pi r_{\mathrm{G}}^{2} \rho_{\infty} v_{\infty}$. This is just an estimate, and for different velocitites it can be different from the actual value by up to a magnitude. Small changes in some parameters can lead to significant changes in others. For example Ikhsanov [9] discussed the value of the critical period which determines the end of the subsonic propeller stage (and thus the beginning of the accretion stage). The obtained value is different from that found in the original paper by Davies and Pringle [6] by a factor of 7.5. Correspondingly all timescales are also significantly changed. However, it should be noted that this is due to a change in the magnetospheric radius $r_{M}$ by a factor of only about 2 !

The second is connected with idealizations. Even if all parameters can be well defined, then it is necessary to take into account such details as the non-spherical form of the magnetosphere, the inclination of the magnetic axis relative to the spin axis, and the angular moment in the infalling matter (even for cases when the condition for disc formation is not fulfilled). For example even if $r_{\mathrm{M}}>r_{\mathrm{c}}$, part of the magnetosphere is inside $r_{\mathrm{c}}$ as the corotation radius is the radius of a cylinder, and not a sphere. In that sense the process of alignment (see, for example, $[10,11])$ during the magnetorotational evolution of an NS can be important in the destiny of an NS.

A low-rate accretion can proceed even at the propeller stage for several reasons. One of these is diffusion of plasma. This form of accretion was discussed in detail by Ikhsanov [7]. For long spin periods, luminosity due to such an accretion can be larger than the dissipation of the rotational energy on the boundary of the magnetosphere.

An important question is connected with the whole time $t_{\mathrm{A}}$ of evolution prior to the accretor stage. Obviously

$$
t_{\mathrm{A}}=t_{\mathrm{E}}+t_{\mathrm{P}}
$$

where $t_{\mathrm{E}}$ is the time which an NS spends in the ejector stage and $t_{\mathrm{P}}$ is the duration of the propeller stage. Even $t_{\mathrm{E}}$ is not well determined. Usually workers assume that spin-down at this stage is determined by the magnetodipole formula with the braking index equal to 3 . However, direct measurement for many radio pulsars show that the braking index is smaller than 3 . Also evolution of the angle between the spin and magnetic axes is usually not taken into account.

As the propeller stage consists of the three substages, therefore it is necessary to write

$$
t_{\mathrm{P}}=t_{\text {super }}+t_{\text {trans }}+t_{\text {sub }}
$$

If $t_{\mathrm{E}}$ and $t_{\mathrm{P}}$ are determined, the fate of NSs for different parameters can be easily shown on the $t_{\mathrm{E}}-t_{\mathrm{P}}$ diagram suggested in [12]. In figure 2 we show an example of such a plot. For this illustration we assume that the accretion regime starts at the period

$$
P_{\mathrm{br}}=4.5 \times 10^{7} \mu_{30}^{16 / 21} \dot{M}_{8}^{-5 / 7} m^{-4 / 21}
$$

where $\dot{M}_{8}=\dot{M} / 10^{8} \mathrm{~g} \mathrm{~s}^{-1}$ and $m=M_{\mathrm{NS}} / M_{\odot}$. Here we renormalize the value given by Ikhsanov [9]. 


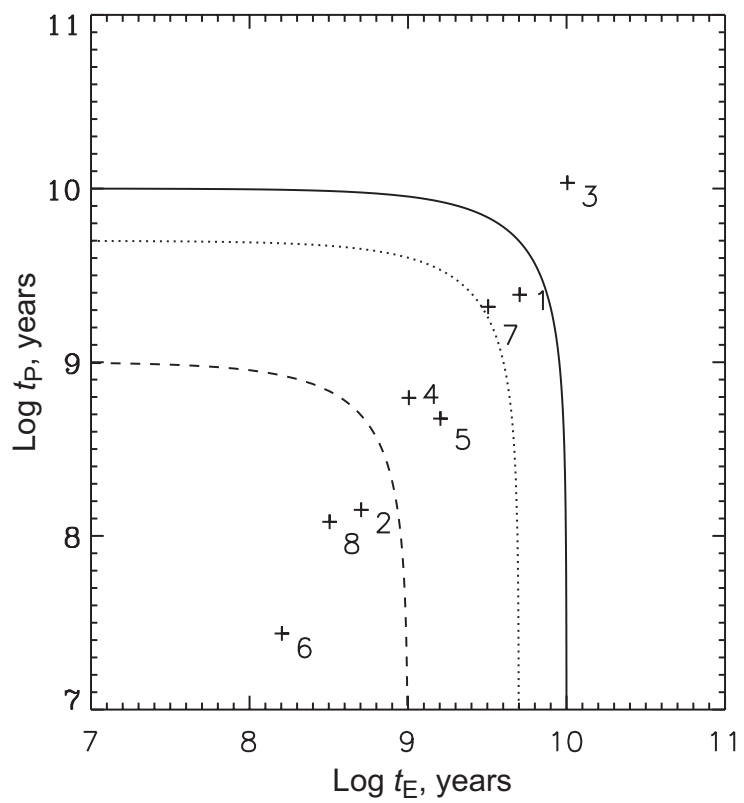

Figure 2. $t_{\mathrm{E}}-t_{\mathrm{P}}$ diagram for isolated NSs. The quasilinear nature of the distribution of the model points on the $\log t_{\mathrm{E}}-\log t_{\mathrm{P}}$ plane was discussed in detail by Popov [12].

Timescales are determined by [12]

$$
t_{\mathrm{E}}=0.8 \times 10^{9} \mu_{30}^{-1} n^{-1 / 2} v_{6} \text { years, }
$$

where $n=\rho m_{\mathrm{p}}^{-1}$ is the interstellar medium number density, and $m_{\mathrm{p}}$ is the proton mass. Also

$$
t_{\text {super }}=1.3 \times 10^{6} \mu_{30}^{-8 / 7} n^{-3 / 7} v_{6}^{9 / 7} \text { years, }
$$

this is a very efficient spin-down suggested by Shakura [13]. So our estimate of $t_{\text {super }}$ is in some sense a low bound:

$$
t_{\text {trans }}+t_{\text {sub }}=10^{3} \mu_{30}^{-2} m P_{\text {br years. }}
$$

The mass of an NS is assumed to be $M_{\mathrm{NS}}=1.4 M_{\odot}$.

In the plot we show lines for $t_{\mathrm{E}}+t_{\mathrm{P}}$ equal to 1,5 and 10 Gyears. The eight numbers corresponds to eight combinations of $n, v$ and $\mu$ (table 1).

Table 1. $t_{\mathrm{E}}$ and $t_{\mathrm{P}}$ for typical values of $n, v$ and $\mu$.

\begin{tabular}{lccrcc}
\hline Number & $n$ & $\begin{array}{c}v \\
\left(\mathrm{~km} \mathrm{~s}^{-1}\right)\end{array}$ & $\mu_{30}$ & $\begin{array}{c}\log t_{\mathrm{E}} \\
\text { (years) }\end{array}$ & $\begin{array}{c}\log t_{\mathrm{P}} \\
\text { (years) }\end{array}$ \\
\hline 1 & 0.1 & 20 & 1 & 9.70 & 9.39 \\
2 & 0.1 & 20 & 10 & 8.70 & 8.15 \\
3 & 0.1 & 40 & 1 & 10.0 & 10.0 \\
4 & 0.1 & 40 & 10 & 9.01 & 8.79 \\
5 & 1.0 & 20 & 1 & 9.20 & 8.68 \\
6 & 1.0 & 20 & 10 & 8.20 & 7.44 \\
7 & 1.0 & 40 & 1 & 9.51 & 9.32 \\
8 & 1.0 & 40 & 10 & 8.51 & 8.08 \\
\hline
\end{tabular}




\section{Conclusions}

In this paper we showed that an intermediate substage of the propeller regime, namely the trans-sonic propeller, should exist. However, the stage is non-stationary and very short. As was shown above, the existence of this intermediate stage does not change the timescale of the evolution prior to the accretor stage (see [7] for a discussion of the timescales). Other conclusions can be summarized as follows.

(1) The intermediate trans-sonic propeller substage is unstable.

(2) The duration of the transition can be roughly estimated as approximately $r_{\mathrm{G}} / v_{\mathrm{ff}}$ (from weeks to years for realistic isolated NSs).

(3) The spin frequency is nearly unchanged during this transition.

(4) The energy release during the transition is small.

\section{Acknowledgements}

The work was partially supported by grants from the Russian Foundation for Basic Research 04-02-16720 and 03-02-16068. S.P. acknowledges a postdoctoral fellowship from the University of Padova where most of this work has been carried out.

\section{References}

[1] V.M. Lipunov, Astrophysics of Neutron Stars (Springer, Berlin, 1992).

[2] V.M. Lipunov, K.A. Postnov and M.E. Prokhorov, Astrophys. Space Phys. Rev. 91 (1996).

[3] V.F. Shvartsman, Izv. Vysshikh Uchebnyk Zavedenii, Radiofizika 131852 (1970).

[4] A.F. Illarionov and R.A. Sunyaev, Astron. Astrophys. 39185 (1975).

[5] R.E. Davies, A.C. Fabian and J.E. Pringle, Mon. Not. R. Astron. Soc. 186779 (1979).

[6] R.E. Davies and J.E. Pringle, Mon. Not. R. Astron. Soc. 196209 (1981).

[7] N.R. Ikhsanov, Astron. Astrophys. 3991147 (2003).

[8] V.M. Lipunov and S.B. Popov, Astron. Rep. 39632 (1995).

[9] N.R. Ikhsanov, Astron. Astrophys. 368 L5 (2001).

[10] T. Regimbau and J.A. de Freitas Pacheco, Astron. Astrophys. 374181 (2001).

[11] V.S. Beskin, A.V. Gurevich and Ya.N. Istomin, Physics of the Pulsar Magnetosphere (Cambridge University Press, Cambridge, 1993).

[12] S.B. Popov, Astron. Astrophys. 418699 (2004).

[13] N.I. Shakura, Soviet Astron. Lett. 1223 (1975). 
